\title{
A COMPARISON OF RTK-GPS VERTICAL COMPONENT WITH PRECISE DIGITAL LEVEL FOR ESTIMATING VOLUMES
}

\author{
Yousif Youkhanna Zaia, Sarhat Mustafa Adam and Sami Mamlook Gilyana \\ Dept. of Surveying Engineering, College of Engineering, University of Duhok, Kurdistan Region-Iraq
}

\begin{abstract}
In this research, RTK GPS (Real time Kinematic GPS) was used to compare with precise Digital Level for estimating earthwork volumes. Any volume calculation process has to pass through two stages. Firstly, data collection using surveying instrument, for instance, level instrument, total station, Global Positioning System (GPS), or laser scanning. In the second stage, carrying out measurement of figuring volumes using either manual or computerized method. In this paper, the field test was conducted to gather data for estimating earthworks using two technologies; Digital Level (DNA 03) and RTK GPS. Two sites have been chosen; open to the sky and partially obstructed to satellites in order to evaluate RTK GPS technique. The main aim is to compare the RTK manufacturer claimed accuracy (height component only) with trusted data. The comparison has been carried out using two different categories; first, through using the volume calculation and second via statistical testing through the cloud to cloud comparison. Finding revealed that the mean differences for the test01 and test02 are about $-20 \mathrm{~mm}$ and $16 \mathrm{~mm}$, respectively. The Root Mean Square (RMS) and maximum error were found to be about $\pm 63 \mathrm{~mm}, 45 \mathrm{~cm}$ and $\pm 56 \mathrm{~mm}, 25 \mathrm{~cm}$ for test01 and test02, respectively.
\end{abstract}

KEYWORDS: Earthworks, RTK GPS, Accuracy, Digital level, Cloud to cloud comparison

\section{1- INTRODUCTION}

I $\mathrm{n}$ engineering family, surveying is one of the main sciences which can determine the positions below and above the surface of the earth using different types of the surveying instrument. Computing volume is one part of engineering surveying which can be used almost in all kinds of constructions and infrastructures such as, buildings, highways, dams, and tunnels. One of the main important engineering works is estimating earthworks. It can have a process of excavating, hauling and placing materials from a location to another using inexpensive methods (Lindeburg, 2012). Usually, compution of earthwork has to pass through two stages which are, gathering data and determination of figuring volumes. Data can be collected using different surveying technologies, for instance, field leveling operations, total station, Global Positioning System (GPS), and laser scanning. These technologies have made the work easier and data can be collected quickly. However, each technology has its advantages and disadvantages.

Earthworks are represented as the highest cost scheme, and the movement of large volumes of earthwork is one of the most significant construction operations (Deakin, 2005). Therefore, it is critical to estimate volumes accurately because in this day and age, in many applications the role of surveying has much consideration to be used with better accuracy. The term of accuracy is common in several applications to express the quality of observations, measurements, and obtained results. Areas and volumes estimation are basic knowledge for most engineering outlines such as route alignment, reservoirs, and tunnels. The excavation and hauling of material on such outlines is the most significant and costly characteristic of the work, on which profit or loss may depend (Schofield, 2001).

Hola and Schabowicz (2010) stated that time, cost and quality when they are combined in any engineering construction, they have to be distributed equally as much as possible to avoid any unforeseen situations. Nowadays, GPS is widely used in construction engineering for computing volumes. Many researchers examined the accuracy of using RTK-GPS in volumes calculation. Shah and Hastak (2008) were used a questionnaire and interview methods to collect data from specialist companies and organizations. From questioner analysis, $30 \mathrm{~mm}(0.1 \mathrm{ft})$ accuracy was achieved in height component.

Another study by Saghravani et al. (2009) showed that the accuracy of RTK-GPS in 
measuring elevation is less than $10 \mathrm{~cm}$. This accuracy was checked by an automatic level which has accuracy of $2.5 \mathrm{~mm} / \mathrm{km}$ and in the area of 16 hectares. However, this accuracy is depended on the situation of satellites in the space. Such accuracy is acceptable in many types of work, for instance, landscaping, installing of power cables and pipelines.

Edwards et al. (2010) examined the accuracy of RTK GPS in their work by taking 6 hours data on six stations. They indicated that the accuracy of using this technique is typically $10-20 \mathrm{~mm}$ in horizontal control and $15-30 \mathrm{~mm}$ in vertical control. Other work by Aponte et al. (2009) confirmed that the accuracy of RTK is within centimeters. However, they also agreed that the GPS static mode could achieve better accuracy than short and long baseline RTK results.

Donald et al. (2007) compared both static and real-time kinematic methods. They showed that the accuracy achieved by RTK was always within centimeters. They agreed that the RTK method is easier than the static mode for obtaining a qualified value for the survey.

In general, RTK GPS gives sub-centimeter accuracy. Nevertheless, this accuracy is depended on how many satellites are available. Also, obstruction of satellite visibility for instant working in an urban canyon, near to tree canopy and tall building can also affect the accuracy of GPS. For earthwork measurements as stated by many authors, this accuracy is adequate and can be used for many engineering applications. Therefore, the likelihood of this paper is to assess and compare Real Time Kinematic (RTK-GPS) manufacturer claimed accuracy (height component only) with trusted data; precise Digital Level DNA03, which have an accuracy of $0.2 \mathrm{~mm}$ over $1 \mathrm{~km}$, for earthwork estimation. For this purpose, two sites were chosen; (open to the sky and partially obstructed to satellites).

\section{2- WORK PLAN AND TESTED AREA}

Two sites were selected to collect data to examine the accuracy of RTK GPS by comparing it with precise Digital Level. One of them was open to the sky, and the other one was partially obstructed to the satellite as shown in Figure1. Two techniques were used to collect data which are GPS and Digital Level. To compute volumes by software, and to compare points cloud to cloud, the three components; Easting, Northing, and Elevation $(\mathrm{E}, \mathrm{N}, \mathrm{H})$ are required to import data to the program. Therefore, as it is known that Digital Level can only compute one element which is elevation, the two other elements, easting, and northing, were measured using GPS. By replacing the elevations that were measured using GPS by elevations of using Digital Level, a complete coordinate of $(\mathrm{E}, \mathrm{N}, \mathrm{H})$ were gathered. The flow chart on Figure 2 shows the overall procedures.

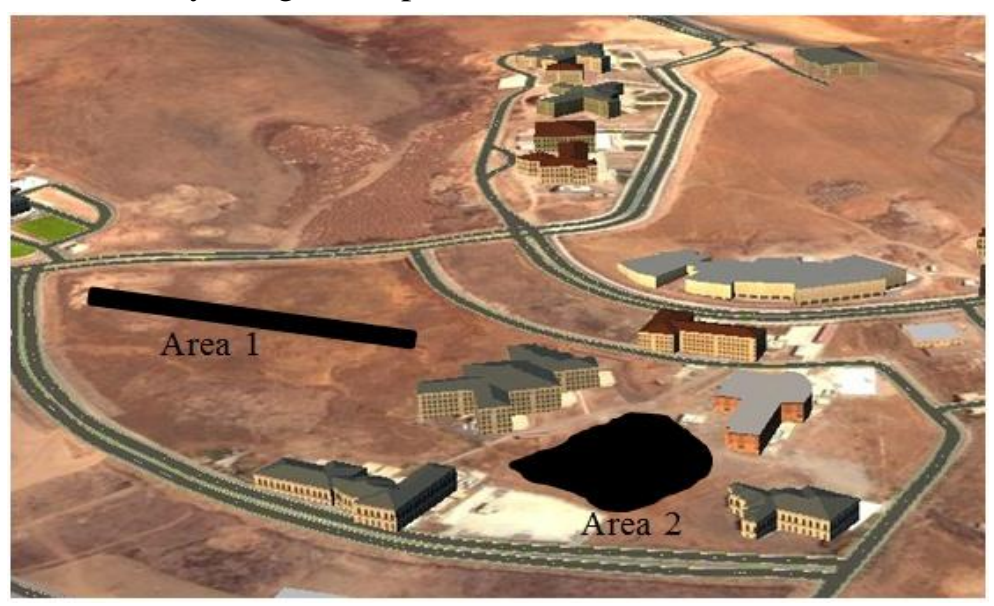

Fig. (1): Experiments' test areas 
Reconnaissance of the two selected areas of study were performed, followed by collecting 200 points and 130 points in tested area 1 and 2 respectively, a sample of collected points shown in Table 1. All gathered data were referenced to a control point C12 (314049.3542, 4080884.034, 508.0934) marker that has been previously surveyed by professional survey team with the dual frequency GPS in static mode. In both sites an alternative changeable plate, for instance, a tile was used to get more accurate results. The survey carried out during morning hours. Both RTK and Leveling surveys were conducted at the same time. The GDOP during observation time was always less than 2 in both tested areas indicating good satellite geometry. Each test took around 3 hours duration of the survey. It is worth mentioning that test01 was a part of 4 th-year graduation project, the survey task was conducted by juniors and their supervisor, whereas experiment02 was carried out by profesional staff.

Table (1): Sample of collected data, open to the sky left, partially obstructed right

\begin{tabular}{|c|c|c|c|c|c|c|c|c|c|}
\hline \multirow[b]{2}{*}{$\#$} & \multicolumn{2}{|c|}{ UTM-WGS84 } & \multicolumn{2}{|c|}{$\begin{array}{l}\text { Elevation (m) } \\
\text { Orthometric }\end{array}$} & \multicolumn{3}{|c|}{ UTM-WGS84 } & \multicolumn{2}{|c|}{$\begin{array}{l}\text { Elevation (m) } \\
\text { Orthometric }\end{array}$} \\
\hline & Easting (m) & Northing (m) & GPS & Digital Level & \# & Easting (m) & $\begin{array}{l}\text { Northing } \\
(\mathrm{m})\end{array}$ & GPS & $\begin{array}{l}\text { Digital } \\
\text { Level }\end{array}$ \\
\hline 1 & 314089.0889 & 4081198.104 & 514.1851 & 514.1507 & 1 & 314128.254 & 4080877.724 & 505.465 & 505.4679 \\
\hline 2 & 314085.9313 & 4081194.226 & 514.057 & 514.0476 & 2 & 314120.8209 & 4080877.314 & 505.5928 & 505.5988 \\
\hline 3 & 314082.8694 & 4081190.287 & 513.9413 & 513.9316 & 3 & 314118.9716 & 4080876.993 & 505.9993 & 506.0226 \\
\hline 4 & 314092.2696 & 4081201.959 & 514.2961 & 514.304 & 4 & 314112.6811 & 4080876.168 & 505.6679 & 505.6863 \\
\hline 5 & 314095.4111 & 4081205.854 & 514.4972 & 514.4826 & 5 & 314112.9475 & 4080869.109 & 505.528 & 505.5487 \\
\hline 6 & 314097.4087 & 4081192.47 & 514.1351 & 514.1468 & 6 & 314117.3936 & 4080869.085 & 505.7087 & 505.7226 \\
\hline 7 & 314094.5696 & 4081188.345 & 513.9666 & 513.9691 & 7 & 314124.1755 & 4080867.062 & 505.4008 & 505.4497 \\
\hline 8 & 314091.7741 & 4081184.196 & 513.7691 & 513.7945 & 8 & 314127.1565 & 4080866.274 & 504.4781 & 504.4875 \\
\hline 10 & 314103.0865 & 4081200.728 & 514.6123 & 514.6256 & 10 & 314138.6724 & 4080866.649 & 505.5808 & 505.5537 \\
\hline
\end{tabular}

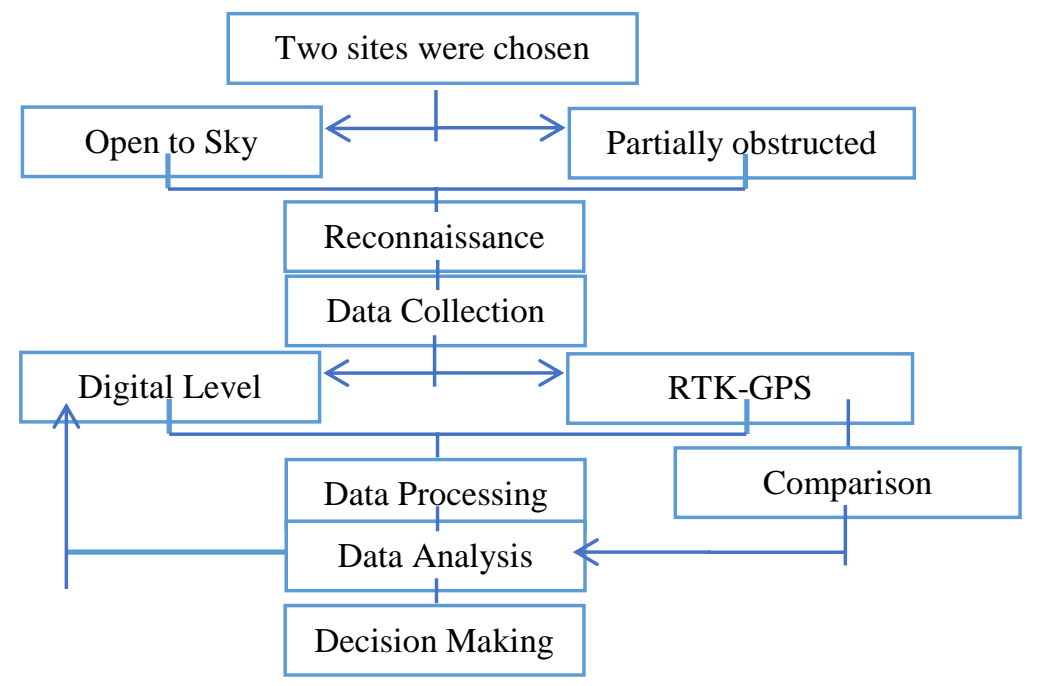

Fig. (2): Procedures or Workflow of the research 


\section{3- RESULT AND DISCUSSION}

Two scenarios will be applied in this paper. On the first scenario, the data from both tools (RTK GPS and Digital Level) will be compared in term of volume calculation. In the second scenario, the data sets will be analyzed further using the cloud to cloud comparison technique.

\subsection{First Scenario: vertical comparison} according to obtained volume

On construction projects, it is often necessary to modify the existing ground levels to create platforms to build on. Accurately calculating the volumes of soil that must be removed (cut) or embanked (fill) to create the final ground levels is an essential part of the planning process (Schofield \& Breach, 2007). As commonly known, there are several different methods used to determine the areas of cut and fill once the sections have been plotted.

Two main methods can be used to calculate volumes which are a manual method and through the use of software method. Nowadays, digital

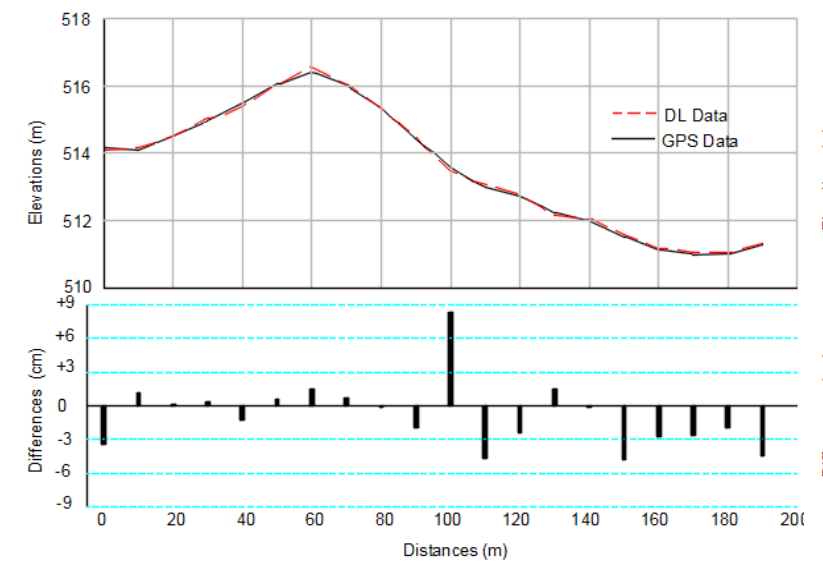

Fig. (3): Profile and differences (Test01 left and Test02 right) methods through special software extensively use than the manual methods. For computing volumes more precisely, AutoCAD Civil 3D from Autodesk was used. After points had been imported into the program, two surfaces were created, and volumes were computed. Figures 3 represent the differences generated from profiles of the two surfaces. The distances between points are the same in both cases (GPS \& DL), and the comparison was done according to the vertical tolerances, and volumes were calculated by subtracting GPS surface with Digital Level. In the test 01 and test 02 , a net of $87 \mathrm{~m} 3$ cut and $84 \mathrm{~m} 3$ fill were computed respectively. As in both tests same instruments were used, it is obvious that in test01 height components for GPS gave both more and less that DL elevations. However, increases in elevations are more than decreases, which is why the test01 gave a net of cut. On the other hand, test 02 gave always decrease which might be related to the fact that more satellite was observed in test 01 than in test02.

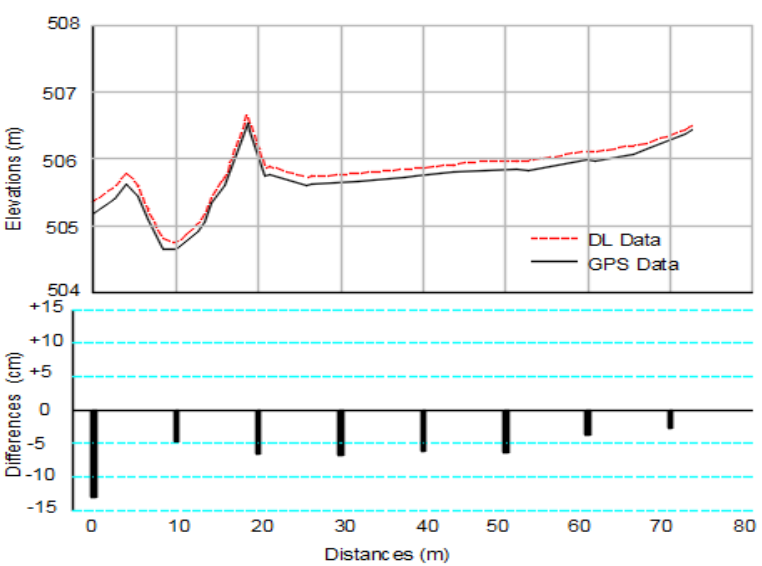

is carried out from compared to the reference. The difference between RTK and DL was calculated for the both tests (test01 and test02). The color visualization of difference on the 3-D models of the both tests is presented on Figure 4. The ramp height or color visualization is ranging from blue for minimum differences to red for the maximum differences. The visual of disagreements on the 3-D mesh allow the user to spot out maximum errors without carrying any further statistical testing.

\subsection{Second Scenario: Cloud to Cloud comparison}

Another scenario to analyze the data sets by looking at the differences with other perspective is through the cloud to cloud comparison using the Cloud Compare $\mathrm{CC}$ free tool. The Cloud Compare CC was used to analyses the differences between the two sets of clouds (RTK and DL). CC allows for absolute distance to be calculated between the two sets of points. One set of data will be used as a compared and other as a reference which comparison or distance measurement 

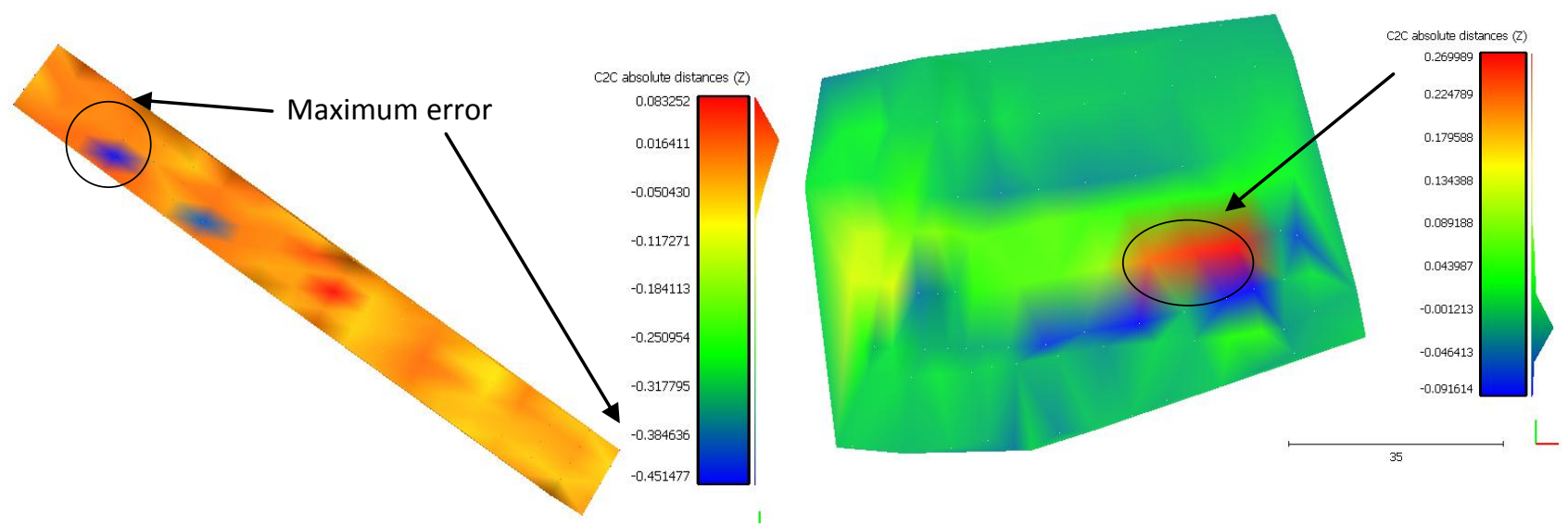

Fig. (4): Color visualization of differences on 3D model (visual height ramp), test01 left, and

As Figure 4 shows, extreme red and blue spots are appearing on both surfaces. These extreme color spots indicate the presence of maximum errors. The rough or approximate values of these differences can be measured from color scale bar on the right of each model. The bar shows the density of the differences located on the blue to green ranges on the right model and in the red to the yellow in left model. These distributions indicate that density of differences is within minimum ranges.
Turning these color visualization to statistical tests is also possible with CC tool. The differences between Digital and GPS in both experimental were analyzed using CC. Histograms of differences were drawn, and Gaussian normal distribution curve was fitted to the histogram differences, Figure 5. The mean of absolute differences can be calculated in addition to the Sigma (RMS) or standard deviation of the differences.
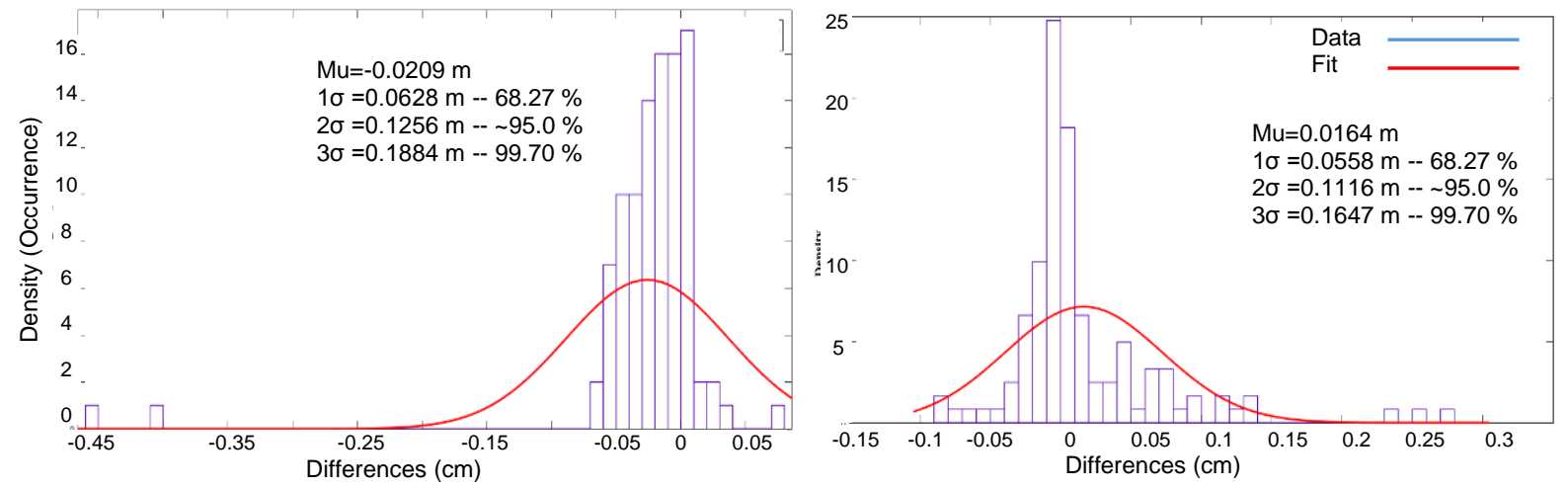

Fig. (5): Histogram differences with Gaussian normal fit, Test01 left and test 02 right

As Figure 5 shows, the mean differences for the test 01 and test 02 are about $-20 \mathrm{~mm}$ and $16 \mathrm{~mm}$, respectively. The Root Mean Square (RMS) or sigma found to be about $63 \mathrm{~mm}$ and $56 \mathrm{~mm}$ for test01 and test02, respectively. One sigma is indicating that $68.3 \%$ of differences are within $\pm 63 \mathrm{~mm}$ and $\pm 56 \mathrm{~mm}$. In term of survey or geomatic's analysis, the $95 \%$ error percentage of data is more frequently use which is about 2 RMS or 2 sigmas. In the first test, the 95 error percentage is equal to about $12 \mathrm{~cm}$ and $11 \mathrm{~cm}$ for the test 02 . The 95 percentage error gives the range of residuals that can be expected to occur $95 \%$ of the time. It is also observed that significant errors can still happen out of $95 \%$ range. The maximum error found to be about $45 \mathrm{~cm}$ and $25 \mathrm{~cm}$ for test 01 and test 02 , respectively. 
Although accuracy is a factor of many parameters, comparing the mean values with Leica Viva manufacturer accuracies show no real differences. According to Leica GS10/15 user manual (Leica Geosystem, 2012), the RTK single baseline error expected for the vertical component is $15 \mathrm{~mm} \pm 1 \mathrm{ppm}$. From Figure 1, knowing that the distance from $\mathrm{C} 12$ to the center of each test area is 285 and $105 \mathrm{~m}$ for test 01 and test 02 respectively. So, the expected error should be in the range of $16 \mathrm{~mm}$ as both baselines are less than $1 \mathrm{~km}$. However, if one compares the RMS values from both tests with the expected RMS value, it can be noticed that both tests show significant differences $\pm 47 \mathrm{~mm}$ and $\pm 30 \mathrm{~mm}$ for test01 and test02 respectively. This significant error could be due to any source of errors or biases, such as satellites availability, multipath error, loss of lock, or an error generated from digital level itself. Although the mean differences are very close in both tests within about $4 \mathrm{~mm}$, interestingly it was found that the average of differences is negative in case of test 01 and positive in case of test 02 . The digital level was set as reference and GPS as data in both experiments. The negative mean differences indicate that the GPS was overestimated the height in case of tes 01 and underestimated in the case of test02.

Despite the fact that test01 is open to sky whereas the test02 is partially obstructed to satellites, test 01 showed that the mean absolute difference is more than test02. It worth to mention that test 01 was conducted by $4^{\text {th }}$-year graduation students whereas test02 was carried out by practice surveyor professional staff. The relative inaccurate results in test 01 could be due to the lack of experience of students in completing the tasks assigned to them, such as holding the staff truly vertical. Alternatively, it could be because the rover in test 02 was very close to the base receiver, baselines ranging from 80 to $130 \mathrm{~m}$.

It is worth mentioning that comparing the time required to complete the RTK GPS in both tests were much lower than the time needed to conduct the level survey, and it was about one-third of the level survey time. Besides, minimum of two people were required to carry out the level field survey; one person should work with the instrument and other holding the staff. However, for RTK-GPS, the task can be only completed with one person. This can help in minimizing the workforce costs by comparing with the digital level.

\section{4- CONCLUSION}

The purpose of this research was to compare RTK GPS height component only with trusted precise data that obtained by accurate digital level $(0.2 \mathrm{~mm}$ accuracy) for computing volumes. Data were gathered using two different techniques as mentioned previously and two main tasks were performed to achieve the objectives of the paper. In the first one, data were collected in an area that opens to the sky, and there were no obstacles to the signals. In the second task, the data gathered were partially obstructed to the signals. The study included the comparison of the RTK-GPS technique with precise digital level for both of the tested areas. The analysis of the results was carried out using two scenarios; comparison in terms of volume computation in the first one, and evaluation in terms of statistical testing using cloud comparison tool.

Finding revealed that the mean differences for the test 01 and test 02 are about $-20 \mathrm{~mm}$ and $16 \mathrm{~mm}$, respectively. The Root Mean Square (RMS) or sigma found to be about $\pm 63 \mathrm{~mm}$ and $\pm 56 \mathrm{~mm}$ for test01 and test02, respectively. Maximum errors were found to be about $45 \mathrm{~cm}$ and $25 \mathrm{~cm}$ for test01 and test02 respectively. Bearing in mind the volume calculation a net of $87 \mathrm{~m}^{3}$ cut and $84 \mathrm{~m}^{3}$ fill were computed for the test01 and test02 respectively. Based on the outcome of the study, GPS has good accuracy, and it can be used for many survey engineering tasks. In spite of GPS can cost extra money than Digital Level, it consumes less time and requires less manpower to gather data. These two points have to be taken into account when selecting an appropriate tool. Therefore, it can be arguably accepted that GPS can be employed for initial surveys, vast areas, and long paths surveys such as road network or pipeline extension. If the project requires high accuracy, Digital Level, on the other hand, can be preferred. Finally, this study suggests that RTK method can be utilized alternatively for the survey leveling process with relatively similar outcome and fewer efforts. 


\section{REFERENCES}

- Aponte, J., Meng, X., Hill, C., Moore, T., Burbidge, M., \& Dodson, A. (2009). Quality assessment of a network-based RTK GPS service in the UK. Journal of Applied Geodesy, 3(1), 25-34.

- Deakin, R. E. (2005). Engineering surveying 1: Earthworks and volumes. Retrieved November 20, 2016, from http://user.gs.rmit.edu.au/rod/files/publications/ Earthworks\%20and\%20Volumes.pdf

- Donald, C., James, Y., \& Ben, S. (2007). Investigation on GPS Heighting Accuracy with the use of Hong Kong Satellite Positioning Reference Station Network (SatRef). Paper presented at the Strategic Integration of Surveying Services, Hong Kong SAR, China.

- Edwards, S., Clarke, P., Penna, N., \& Goebell, S. (2010). An examination of network RTK GPS services in Great Britain. Survey Review, 42(316), 107-121.

- Hola, B., \& Schabowicz, K. (2010). Estimation of earthworks execution time cost by means of artificial neural networks. Automation in Construction, 19(5), 570-579.
- Leica Geosystem. (2012). Leica GS10/GS15 User Manual Retrieved February 10, 2017, from http://www.surveyequipment.com/PDFs/Leica Viva_GS10_GS15_User_Manual.pdf

- Lindeburg, M. R. (2012). Civil engineering reference manual for the PE exam: www. ppi2pass. com.

- Saghravani, S. R., Mustapha, S. b., Saghravani, S. F., Ibrahim, S. b., \& Yusoff, M. K. b. (2009, 3-5 April 2009). Performance of Real-Time Kinematic Global Positioning System and Automatic Level Surveying for Height Determination - A Comparison. Paper presented at the 2009 International Conference on Signal Acquisition and Processing.

- Schofield, W. (2001). Engineering Surveying: Theory and Examination Problems for Students (5th ed.). Oxford: Butterworth-Heinemann.

- Schofield, W., \& Breach, M. (2007). Engineering Surveying (6th ed.). Oxford: ButterworthHeinemann.

- Shah, S., \& Hastak, M. (2008). Acquistion and Analysis of Earthwork Pay Quantity Verification Daya. Joint Transportation Research Program, 317. 